\title{
Scalar leptoquarks and Higgs pair production at the LHC
}

\section{Tsedenbaljir Enkhbat}

Elementary Particle and Nuclear Research Group, Institute of Physics 8 Technology, Mongolian Academy of Sciences, Ulaanbaatar 13330, Mongolia

Department of Physics and Center for Theoretical Sciences, National Taiwan University, Taipei 10617, Taiwan

E-mail: enkhbat@gmail.com

ABSTRACT: The presence of colored particles can affect both the single and the pair Higgs productions substantially. For scalar particles, this happens if their portal couplings to the Standard Model Higgs are large and their masses are not too high. In the present work these processes are studied in the case of several leptoquarks which may appear in many beyond Standard Model theories. It is found that the constraints on the portal couplings from the single Higgs production and the decays to various channels measured by the LHC experiments still allow increased Higgs pair production rate. For the masses in the range from $180 \mathrm{GeV}$ to $300 \mathrm{GeV}$, depending on the strength of such portal couplings, the Higgs pair production may reach an order to several hundred in magnitude larger rate than the Standard Model case for the $8 \mathrm{TeV}$ run. Therefore, combined with the on going searches for leptoquarks by both the CMS and ATLAS, this is one of the possible scenarios to be probed directly by the current data. The current study demonstrates that if colored scalars modify scalar potentials through portal couplings, which has been studied for variety of motivations such as playing a potentially important role in electroweak phase transition, composite models or radiative neutrino masses, this fact may appear as the modified Higgs pair production.

KeYwords: Higgs Physics, Beyond Standard Model

ARXIV EPRINT: 1311.4445 


\section{Contents}

1 Introduction 1

2 Light LeptoQuarks 2

3 Higgs phenomenology with light LeptoQuarks 4

4 Conclusions and discussions $\quad 10$

$\begin{array}{ll}\text { A Amplitudes } & 11\end{array}$

\section{Introduction}

The data collected by the LHC experiments at 7 and $8 \mathrm{TeV}$ with $\sim 5$ and $20 \mathrm{fb}^{-1}$ respectively is refining the details of the Higgs like resonances found last year [1,2]. Many decay channels have been searched for and the individual channels so far have given us a consistent picture with what one expects from the SM Higgs. On the other hand, the self interaction of the Higgs, which is probed by the Higgs pair production [3-7], is too feeble in the SM to be detected with these early data set. Even at $14 \mathrm{TeV}$ run, the luminosity required for probing this process is very high [7-18]. This fact, namely the smallness of the corresponding Higgs pair production cross-section, makes it prone to a presence of a new physics [19-33].

In particular, relatively light colored particles are known to affect the cross-section substantially [19-24]. As a mater of fact there are many models with various motivations including models of GUT remnants [34-41], composite models [42-50] or in a radiative neutrino mass models [51-53] which may give such contributions. Among these the scalars are interesting as they may play crucial role in the spontaneous symmetry breaking through additional terms with large portal couplings in the scalar potential. Furthermore, another reason to be interested in colored scalars is that they are known to have a potentially crucial role on achieving a successful electroweak phase transition (EWPT). Common feature of these models is that the colored particle(s) must be light enough for a strong enough EWPT [54-62]. The discovery of the new resonance has triggered renewed interest in colored particles from this point of view and several groups have made detailed studies. Multiple scalars tend to broaden available parameter space for EWPT. For example, the so called light stop scenario has been the subject of a recent study [60,62]. Due to their possible importance it is crucial to study more broader class of models with colored scalars.

In the present work we study the phenomenological consequences of the Standard Model extension by two or more colored scalar particles. As a case study we take several leptoquarks (LQ) since there is an active experimental program by both ATLAS and CMS for the search [63-69]. The LHC search for an individual LQ have now reached as high 
as $830 \mathrm{GeV}, 525 \mathrm{GeV}$ with $5 \mathrm{fb}^{-1} 7 \mathrm{TeV}$ data for first and third generation LQs, $1070 \mathrm{GeV}$ with $20 \mathrm{fb}^{-1} 8 \mathrm{TeV}$ data for second generation LQ respectively assuming they decay $100 \%$ to the considered decay channels. If the LQ masses are above these limits, their effect on the Higgs phenomenology would be very minimal. On the other hand simultaneous presence of several LQs, may open up additional channels and therefore weakens these bounds. Specific models where the LQs are introduced to explain a certain phenomenon usually requires more than one LQs as in the model we study here.

I examine a possibility of the existence of LQs with masses as light as $\sim 200 \mathrm{GeV}$ and study their effect for the single and di Higgs productions. As we will see the Higgs pair production is substantially altered in the low mass range below $300 \mathrm{GeV}$ without too much change in the Higss diphoton decay channel if portal couplings are large. These couplings are required to have opposite signs by the latest Higgs data or small in magnitude. The model I consider has two LQs, an $\mathrm{SU}(2)$ doublet $\omega$ and a singlet $\chi$. As we will see their simultaneous presence still allows them to have relatively light masses and escape the current bounds. In particular, the current bounds do not include LQs decaying to $\mu t$ or $\tau t$. Such a scenario, for example, has appeared in a model considered by Babu and Julio [51], where the light neutrino masses are induced by two-loop effects from LQs. If their masses are only of order few hundred $\mathrm{GeV}$, as it is required in this case, the scenario can be probed or even excluded with the data from the LHC. Therefore this is one of the easiest model which can be tested and is the subject of the current study. Although I consider a particular model, it should be stressed that other models with colored particles can affect the pair productions in a similar manner.

In section 2, I briefly list the current experimental status on the Higgs production and decay rates. Then I introduce the model I examined in the paper. Section 3 contains main part of this work where the numerical results for the single and pair Higgs productions are presented. The conclusion is given in section 4 .

\section{Light LeptoQuarks}

ATLAS and CMS both have released their results on the Higgs searches from 7 and $8 \mathrm{TeV}$ runs. The median significance of the diphoton channel for ATLAS, while remains above the SM level, has come down to $\mu_{\gamma \gamma}=1.53_{-0.3}^{+0.34}[70]$ compared to the $7 \mathrm{TeV}$ result. On the other hand the change in the latest CMS result compared to its $7 \mathrm{TeV}$ data was more dramatic. Depending on the analysis the signal strength now stands either at $\mu_{\gamma \gamma}=0.78_{-0.26}^{+0.28}$ or $1.11_{-0.30}^{+0.32}$ [71]. Also importantly, the measurements for $h \rightarrow Z Z^{*} \rightarrow 4 \ell$ channel strength are $\mu_{4 \ell}=0.91_{-0.24}^{+0.30}$ from CMS [72] and $\mu_{4 \ell}=1.7_{-0.4}^{+0.5}$ [70] from ATLAS respectively which constrain the production separately. These results indicate that the diphoton channel of Higgs decay is closer to the SM prediction than it has appeared from the $7 \mathrm{TeV}$ data. Therefore, any new resonance should not affect the single Higgs production and the diphoton channel too much. This requirement alone makes a single colored scalar object harder to exist at lower mass range if its portal coupling of $|H|^{2}|X|^{2}$ type is large. If such couplings are small they will not play any interesting role in the Higgs phenomenology. On the other hand several colored scalars can lead to interesting excesses that may be checked with the existing data at the same time satisfying various Higgs decay channels measurements. 
The model I examine in this paper contains two new multiplets, $\mathrm{SU}(2)_{L}$ singlet and doublet scalar leptoquarks $\Omega \sim(3,2,1 / 6)$ and $\chi \sim(3,1,-1 / 3)$ [51]. The Lagrangian of the model is given as:

$$
\begin{aligned}
\mathcal{L}= & \left(Y_{i j} \Omega i \sigma_{2} L_{i} d_{j}^{c}+F_{i j} \chi e_{i}^{c} u_{j}^{c}-\mu \Omega^{\dagger} H \chi+\text { h.c }\right)-m_{\Omega}^{2}|\Omega|^{2}-m_{\chi}^{2}|\chi|^{2} \\
& -\lambda_{\omega}|\Omega|^{2}|H|^{2}-\lambda_{\chi}|\chi|^{2}|H|^{2}-\kappa\left|\Omega^{\dagger} H\right|^{2} .
\end{aligned}
$$

After electroweak symmetry breaking, the lower component of the doublet LQ will mix with the singlet $L Q$ via the trilinear $\mu$-term which we denote as $\chi_{1}$ and $\chi_{2}$, and the remaining upper $2 / 3$ charged component as $\omega$. Their physical masses are given by

$$
\begin{aligned}
m_{\omega}^{2} & =m_{\Omega}^{2}+\frac{\lambda_{\omega}}{2} v^{2} \\
m_{\chi_{1}, \chi_{2}}^{2} & =\frac{1}{2}\left(m_{\omega}^{2}+\frac{\kappa}{2} v^{2}+m_{\chi}^{2}+\frac{\lambda_{\chi}}{2} v^{2} \mp \sqrt{m_{\omega}^{2}+\frac{\kappa}{2} v^{2}-m_{\chi}^{2}-\frac{\lambda_{\chi}}{2} v^{2}+2 \mu^{2} v^{2}}\right), \\
\tan 2 \vartheta & =\frac{2 \sqrt{2} \mu v}{2 m_{\omega}^{2}+\kappa v^{2}-2 m_{\chi}^{2}-\lambda_{\chi} v^{2}},
\end{aligned}
$$

where $\vartheta$ and $m_{\chi_{1}, \chi_{2}}$ are the mixing angle and masses for the $-1 / 3$ charged LQs $\chi_{1}$ and $\chi_{2}$. $m_{\omega}$ is the mass of $2 / 3$ charged component denoted as $\omega$. This spectrum was proposed by Babu and Julio as an explanation for the light neutrino masses induced by two-loop effects of the LQs. Readers interested in are referred to the original paper where exhaustive list of many flavor implications were discussed. Several scenarios in the model requires these LQs to be lighter than $500 \mathrm{GeV}$, which makes them testable at the LHC. I concentrate primarily on the portal couplings and study their collider aspect and examine the consequences.

The searches for LQs at LHC have given lower bounds on their masses for several different LQ decay channels for the data collected at $7 \mathrm{TeV}$ by both CMS and ATLAS collaborations. Assuming $100 \%$ branching fraction to electron or muon with a light quark, the pair produced LQs decaying to two leptons of the same flavor with at least two jets or single lepton with missing transverse energy and two jets have been ruled out up to 830 [63] with $7 \mathrm{TeV}$ data and $1070 \mathrm{GeV}$ [64] with full data for electron and muon channels respectively at $95 \%$ confidence level by CMS collaboration. If the branching fractions are assumed to be $50 \%$ the limits are 630 and $840 \mathrm{GeV}$ respectively. The third generation LQs are ruled out up to $450 \mathrm{GeV}$ for $\nu \bar{b}$ by CMS [65], and 525 and $535 \mathrm{GeV}$ for $b \tau$ by CMS [66] and ATLAS [67] respectively. For the $b \tau$ channel the bound from CMS weakens to $\sim 230 \mathrm{GeV}$ if the branching fraction is $\sim 60 \%$. The ATLAS collaboration has not updated their searches for lighter generation LQs $[68,69]$ beyond $7 \mathrm{TeV} 1 \mathrm{fb}^{-1}$ data set. A thorough collider search analysis is beyond scope of this paper. Interested readers are referred to refs. [48, 73-76]. In spite of all the above experimental advances in various channels, the searches for LQs decaying to $\mu t$ or $\tau t$ have not been done.

If one considers any of the LQs, the LHC searches require that their masses have to be above $450 \mathrm{GeV}$. Unless corresponding portal coupling is very large the both single and di Higgs productions will not be affected at any interesting level. In the following we explain that these constraints may not be applicable for the model given by the Lagrangian in 
eq. (2.1), To do so we consider a case where the following mass hierarchy holds: $m_{\omega}>$ $m_{\chi_{2}}>m_{\chi_{1}}$. If the couplings $Y_{i j}$ in Eq. (2.1) are small enough such that the mass splitting between $2 / 3$ and $-1 / 3$ charged LQ makes $\omega \rightarrow \chi_{i} W^{+*} \rightarrow \chi_{i} \bar{f}_{d} f_{u}$ channel dominant, these bounds are evaded. We call these three-body channels. Here $\bar{f}_{d} f_{u}=(\bar{d} u, \bar{s} c, \bar{\ell} \nu)$. The star signifies that the $W$ is off-shell. This is because the electroweak precision test requires the mass splitting within the $\mathrm{SU}(2)_{L}$ doublet components be less than $\sim 52 \mathrm{GeV}$ [77].

Both the ATLAS and CMS have put the constraint on the mass by varying the branching fractions of the searched channels. ATLAS puts $\sim 10 \%$ and $5 \%$ upper bounds on the branching fraction for LQ decaying to $\mu q$ only and decaying equally to $\mu q$ and $\nu q$ respectively for LQ mass $200 \mathrm{GeV}$. The CMS has similar results but only down to $250 \mathrm{GeV}$ Nevertheless, with larger data set of $5 \mathrm{fb}^{-1}$, the branching fractions to eejj, e $\nu j j, \mu \mu j j$ and $\mu \nu j j$ for LQ pair are constrained to be below $\sim 12,2,12$ and 2 percents respectively. The new data set from $8 \mathrm{TeV}$ will surely strenthen these further. In the scenario we consider the most stringent constraint comes from $\omega^{2 / 3} \rightarrow \ell q$ searches. As for the $b \tau$ and $b \nu_{\tau}$ channels the branching fractions have to be below $\sim 26 \%$ and $\sim 60 \%$ respectively. To avoid these we assume the corresponding branching fraction be less than $\sim 10 \%$ that of the three body channel. As long as we choose values small enough for $Y_{i j}$ satisfying the above inequality, the constraints from the searches for the light flavor LQs are avoided. Among the $F_{i j}$ couplings only $F_{23}$ and $F_{33}$ are allowed to be large by the LQ searches since they lead to the not-yet-searched $t \mu$ and $t \tau$ decay channels. Therefore we further assume the other $F_{i j}$ couplings are small and satisfy the constraints from various flavor changing neutral current constraints $[51,52,78,79]$. Further, if $F_{23}$ and/or $F_{33}$ are the largest $F_{i j}$ couplings, $\chi \rightarrow \mu \bar{t}$ or to $\tau \bar{t}$ will be the dominant $\chi_{i}$ decay channel. The experimental bound $\tau \rightarrow \mu \gamma<4.4 \times 10^{-8}$ puts constraint $\left|F_{23} F_{33}^{*}\right| \lesssim 0.2 \times\left(m_{1} / 200 \mathrm{GeV}\right)^{2}$ which allows even a value of order one for either of these couplings. From the above discussion we see that the signals for the $\omega$ pair production are $\chi_{i} \chi_{j} W^{+*} W^{-*}$ with the off shell $W^{\prime}$ 's subsequently decaying either hadronically or leptonically when $F_{23}$ or $F_{33}$ is the largest coupling.

\section{Higgs phenomenology with light LeptoQuarks}

In the last section we have argued that the current limits from the LHC experiments still allow LQs with light masses down to $\sim 180 \mathrm{GeV}$. Given that such a possibility exists in the current section we study their phenomenological consequences. The possibility that the Standard Model Higgs could have portal couplings to an unknown sector has been a subject of many studies due to its possible role in the electroweak symmetry breaking, electroweak phase transition and as the contact with the dark sector. Recent discovery of the SM Higgs like resonance has intensified such studies.

The effect we investigate here is the Higgs boson pair production. We take several LQs and choose large portal couplings to demonstrate the di-Higgs production rate can be dramatically increased while the single Higgs production and diphoton rates are affected within the experimentally measured values. This will happen even with the current data if the LQs are relatively light below $300 \mathrm{GeV}$, which makes the model testable in most of the considered mass range. 
From the Lagrangian given in eq. (2.1), the LQ and Higgs interactions are easily written down in the mass eigenstates as follows

$$
\begin{aligned}
V_{\mathrm{LQ}-\mathrm{h}}= & \left\{\left(\lambda_{\omega} c_{\vartheta}^{2}+\kappa c_{\vartheta}^{2}+\lambda_{\chi} s_{\vartheta}^{2}\right)\left|\chi_{1}\right|^{2}+\left(\lambda_{\omega} s_{\vartheta}^{2}+\kappa s_{\vartheta}^{2}+\lambda_{\chi} c_{\vartheta}^{2}\right)\left|\chi_{2}\right|^{2}\right. \\
& \left.+\lambda_{\omega}|\omega|^{2}+\left(\lambda_{\omega}+\kappa-\lambda_{\chi}\right) s_{\vartheta} c_{\vartheta}\left(\chi_{1} \chi_{2}^{*}+\chi_{2} \chi_{1}^{*}\right)\right\}\left(\frac{h^{2}}{2}+h v\right) \\
& +\left\{\mu\left(\left(\left|\chi_{2}\right|^{2}-\left|\chi_{1}\right|^{2}\right) c_{\vartheta} s_{\vartheta}+\chi_{1}^{*} \chi_{2} c_{\vartheta}^{2}-\chi_{2}^{*} \chi_{1} s_{\vartheta}^{2}\right)+\text { h.c }\right\} \frac{h}{\sqrt{2}},
\end{aligned}
$$

where $s_{\vartheta}\left(c_{\vartheta}\right) \equiv \sin \vartheta(\cos \vartheta)$. We choose the physical masses $m_{\omega, \chi_{(1,2)}}$, portal couplings $\lambda_{\omega}$, $\lambda_{\chi}$ and the mixing angle as the input parameters. Then the remaining parameters $\mu$ and $\kappa$ are fixed through eqs. (2.2)-(2.4).

The leading order (LO) partonic amplitude for Higgs productions cross-section and the diphoton decay rates are given by:

$$
\begin{aligned}
\sigma_{g g \rightarrow h} & =\frac{G_{F} \alpha_{s}^{2}}{126 \sqrt{2} \pi}\left|\frac{1}{2} A_{\frac{1}{2}}\left(x_{t}\right)+\sum_{i} C_{i} \frac{\lambda_{i} v^{2}}{4 m_{s_{i}}^{2}} A_{0}\left(x_{s_{i}}\right)\right|^{2}, \\
\Gamma_{\gamma \gamma} & =\frac{G_{F} \alpha^{2} m_{h}^{3}}{126 \sqrt{2} \pi^{3}}\left|A_{1}\left(x_{W}\right)+\frac{4}{3} A_{\frac{1}{2}}\left(x_{t}\right)+\sum_{i} \frac{\lambda_{i}}{g_{w}} \frac{m_{W}^{2}}{m_{s_{i}}^{2}} d_{i} Q_{i}^{2} A_{0}\left(x_{s_{i}}\right)\right|^{2},
\end{aligned}
$$

where $x_{\phi}=4 m_{\phi}^{2} / m_{h}^{2}$ for $\phi=t, s_{i}, W$ and the well known loop functions are listed $A_{\left(0, \frac{1}{2}, 1\right)}$ in the appendix. The NLO and NNLO corrections are substantial leading to an enhancement of $K \sim 2$ [7-18]. Since we are primarily interested in the changes from the additional states we take the ratio of the new rates compared and that of the SM where the NLO and NNLO corrections are expected to largely cancel out. The values of the loop functions for $W$ and top are $A_{1}\left(x_{W}\right)=-8.3$ and $A_{1 / 2}=1.38$.

In the Standard Model contributions from the top quark triangle and box diagrams largely cancel each other for $\sim 125 \mathrm{GeV}$ Higgs mass resulting in a few fb production cross section. It is estimated that with few thousand $\mathrm{fb}^{-1}$ at $14 \mathrm{TeV}$, a $3 \sigma$ evidence may be reached [7-18]. This situation may be altered by additional colored particles. The parton level cross-section is given by

$$
\frac{d \hat{\sigma}_{g g \rightarrow h h}}{d \hat{t}}=\frac{G_{F}^{2} \alpha_{s}^{2}}{256(2 \pi)^{3}}\left(\left|\frac{3 m_{h}^{2}}{\hat{s}-m_{h}^{2}+i m_{h} \Gamma_{h}} F_{t r i}+F_{\mathrm{box}}\right|^{2}+\left|G_{\mathrm{box}}\right|^{2}\right) .
$$

There are two types of amplitudes, $F$ and $G$, corresponding to the same and opposite polarization of the incoming gluons respectively. The same polarization part comes from triangular and box diagrams while the opposite one does only from box diagrams. Here the triangular is meant to be the one with the Higgs propagator and therefore is proportional to the Higgs self coupling. The other triangle diagrams not proportional to the Higgs self coupling are combined with the box diagrams. The amplitudes in the SM and in models with additional colored scalars are given in the appendix.

For the masses we take hierarchy $m_{\omega}>m_{\chi_{2}}>m_{\chi_{1}}$. In addition I choose $\Delta m \equiv$ $m_{\omega}-m_{\chi_{2}}=10$ and $50 \mathrm{GeV}$ for small and large splitting and a constant value of $10 \mathrm{GeV}$ for the mass splitting between the lighter two $m_{\chi_{2}}-m_{\chi_{1}}=10 \mathrm{GeV}$. I take two different values for the $\mathrm{LQ}$ mixing $\sin \vartheta=0.1$ and $1 / \sqrt{2}$ for small and large mixings respectively. 
Previous studies have considered an effect of a single colored particles, where one is forced to have a specific couplings not to upset the Higgs production rate. For example, the new physics contribution is chosen to be roughly twice larger and opposite in sign to have unaltered rate. This inevitably affects diphoton channel. In particular among possible color scalars only octet candidate was a good choice [21]. For these models, stability of vacuum requires increasingly stronger portal couplings as the mass is increased [80]. This is because one needs to keep the new contribution to the Higgs production more or less constant for higher mass values which is possible only if the corresponding portal coupling is simultaneously increased. This is not required in our case, since we have several new contributions which can be kept under control by a judicious choices of the various portal couplings as far as the Higgs production and diphoton channels are concerned.

We first scan over the $\lambda_{\omega}$ and $\lambda_{\chi}$ parameter space for the Higgs pair production and super impose the allowed regions by both CMS and ATLAS experiments by the diphoton and $Z Z^{*}$ channels. The result is show in figure 1. The lightest LQ mass is chosen to be $m_{\chi_{1}}=200 \mathrm{GeV}$. The parameter scan has been done using MadGraph 5 [81] with CTEQ6L1 PDF set [82]. The Madgraph implementation of the Higgs pair production in the SM has been modified to include contributions from the LQ. The code has been checked against previously known results such as in ref. [21] and was found to be in an excellent agreement.

As we can see there are regions in the parameter space where the single Higgs production and decay rates are compatible with either of CMS and ATLAS experiments. Depending on the values for the couplings the Higgs pair production may become substantially enhanced. The shape of the regions are easily understood. The single Higgs production rate and decay to diphoton and $Z Z^{*}$ channels will be affected less if the contributions from the LQs largely cancel each other. This fact is reflected in the stripe regions. There is another possibility that the total LQ contribution is twice bigger than the SM amplitude and but opposite in sign as has been done in refs. [21, 22]. This possibility is represented by the allowed region in the lower right corner of the scanned plots in figure 1 where both $\lambda_{\omega}$ and $\lambda_{\chi}$ are large and negative. Since this region will be pushed to higher values as the LQ mass is increased we do not consider this region further and concentrate on the stripe regions.

While these regions obviously should become larger for heavier choice of the LQ mass $m_{\chi_{1}}$, to make sure that the allowed parameters from the scanning are not accidental for the particular choice I made, the single Higgs production is calculated for several set of $\lambda_{\omega}$ and $\lambda_{\chi}$ with $m_{\chi_{1}}$ changing from 180 to $300 \mathrm{GeV}$. The results are plotted and shown in figure 2 . The plots in the right column labeled as $R(g g \rightarrow h)$ are the single Higgs production rate and the plots in the left column are for the corresponding signal significance in the diphoton channel labeled as $\mu_{\gamma \gamma}$ both compared to the SM. As we see the rates are within the one $\sigma$ range of the either of the two experiments at the LHC and approach to the SM values with increasing mass as one would expect.

Next, I estimate the Higgs pair production for the same set of parameters. The results are shown in figures 3 and 4. These are the main results of the present work. As we can see the rate may be enhanced quite substantially compared to the SM expectation even the single Higgs production is affected moderately. The cancellation due to the opposite sign 

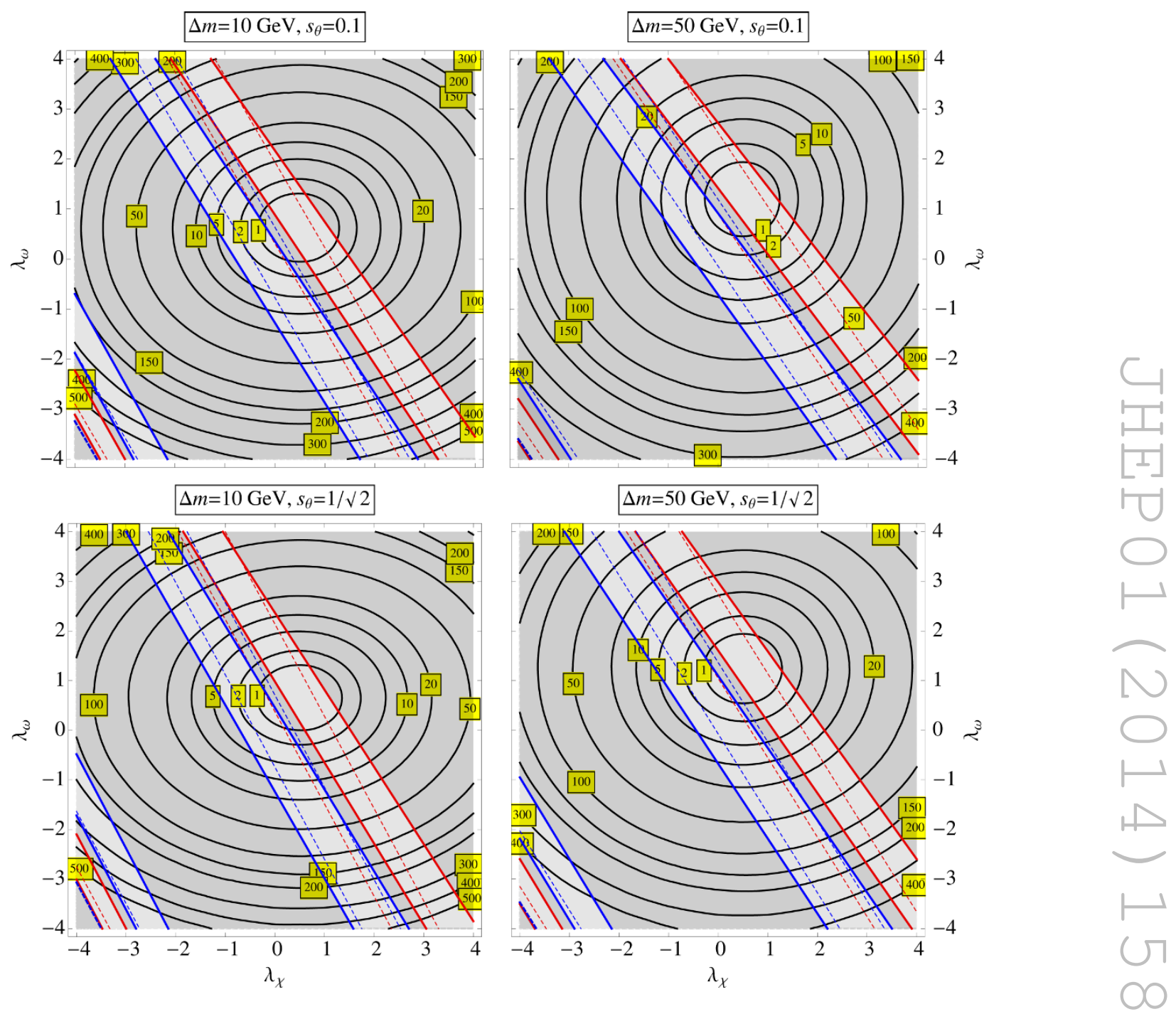

Figure 1. Scanned contour plot in $\lambda_{\omega}-\lambda_{\chi}$ for the ratio Higgs pair productions due LQs and the SM. Here the mass of the lightest LQ is chosen to be $200 \mathrm{GeV}$.

for $\lambda_{\omega}$ and $\lambda_{\chi}$, which kept the single Higgs rate largely unchanged, is still operational for the triangular loop diagram contributions to the pair production. However, there are diagrams quadratic in the portal couplings whenever the final state Higgses come from different vertices. They will contribute constructively even if the single Higgs production remain the same as in the SM. The largest values I chose for the portal couplings require even larger value for the quartic couplings for LQ to make the vacuum at least metastable [80] since we have a negative portal coupling. If we generously allow and take values up to $4 \pi$ for the quartic couplings the metastability of the vacuum is guaranteed.

A detailed signal simulation for the LQ pair productions for the LHC experiments is beyond the scope of the present paper. Nevertheless, few comments are in order. The search for $p p \rightarrow L Q \bar{L} Q \rightarrow t \tau^{-} \bar{t} \tau^{+}$signal has not been done by either of the two collaborations. The pair production cross-section is roughly an order of magnitude below that of $t \bar{t}$ if $m_{L Q}$ little above $m_{t}$. Then the signal is hard to distinguished from $t \bar{t}$ as the taus would not be 

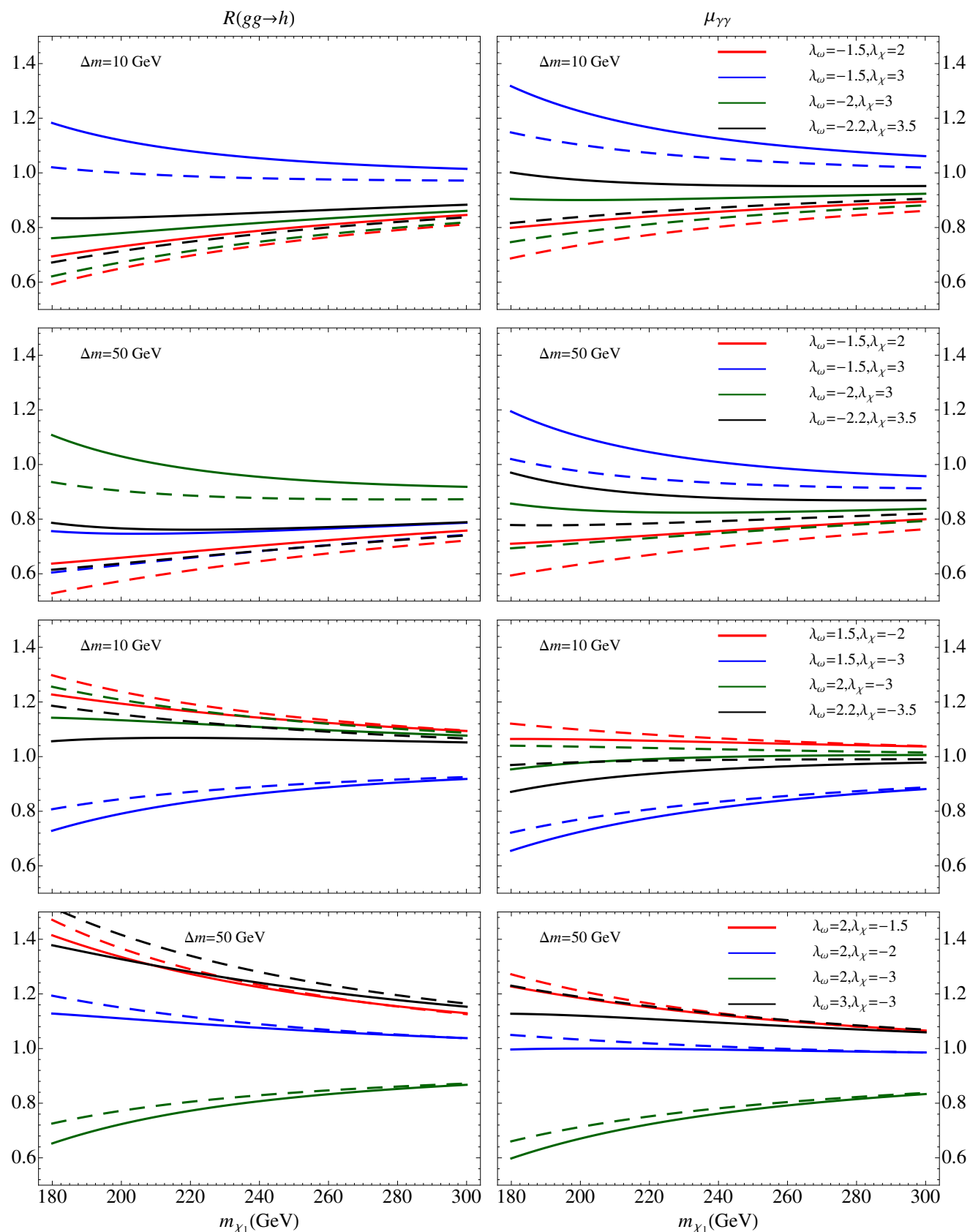

Figure 2. The Higgs production rate and its significance in the presence of several LQs compared to the SM. The solid (dashed) curves are for the LQ mixing angle with $\sin \theta_{\chi}=0.1(1 / \sqrt{2})$.

energetic enough. Therefore such light LQs are still a possibility. For higher values starting around $200 \mathrm{GeV}$ and upto $\sim 260 \mathrm{GeV}$, recently performed searches for the Higgs production in association with a top pair $g g \rightarrow t \bar{t} h$, with Higgs decaying to tau pair [83, 84], may rule out some mass regions whenever the chosen cuts are applicable to the event generated by the LQ pairs. This process has the same final state as the pair produced leptoquarks decaying to $t \tau$. The observed upper bound is $\sigma / \sigma_{S M}=13$ with signal strength $\mu=-0.7_{-5.3}^{+6.2}$. 


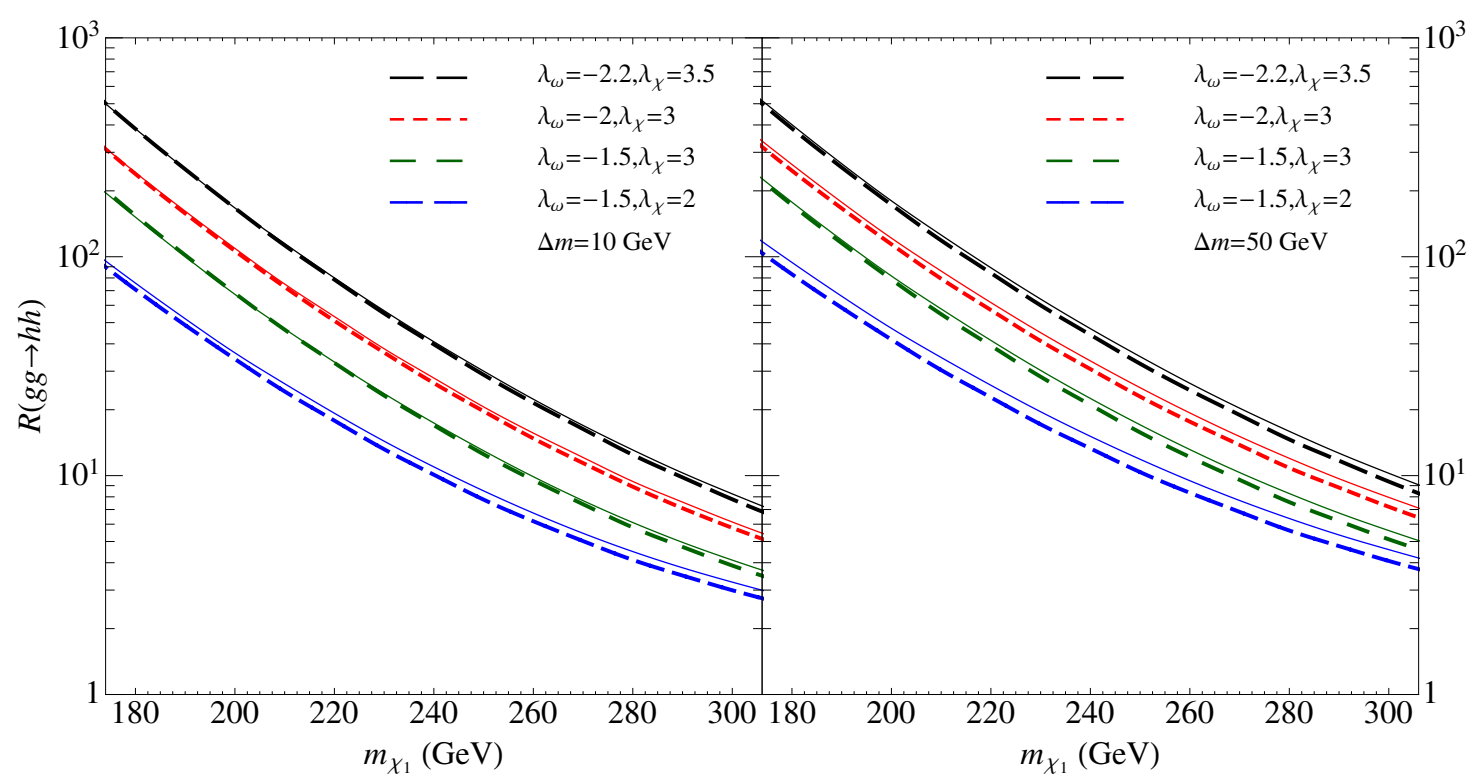

Figure 3. The ratio of Higgs productions due LQs and the SM for negative $\lambda_{\omega}$ and positive $\lambda_{\chi}$ for several different choices for the mixing parameter $s_{\theta}=0.1$. Thin lines with the same colors to the various dashed lines are obtained when the maximal mixing $s_{\theta}=1 / \sqrt{2}$ is chosen.

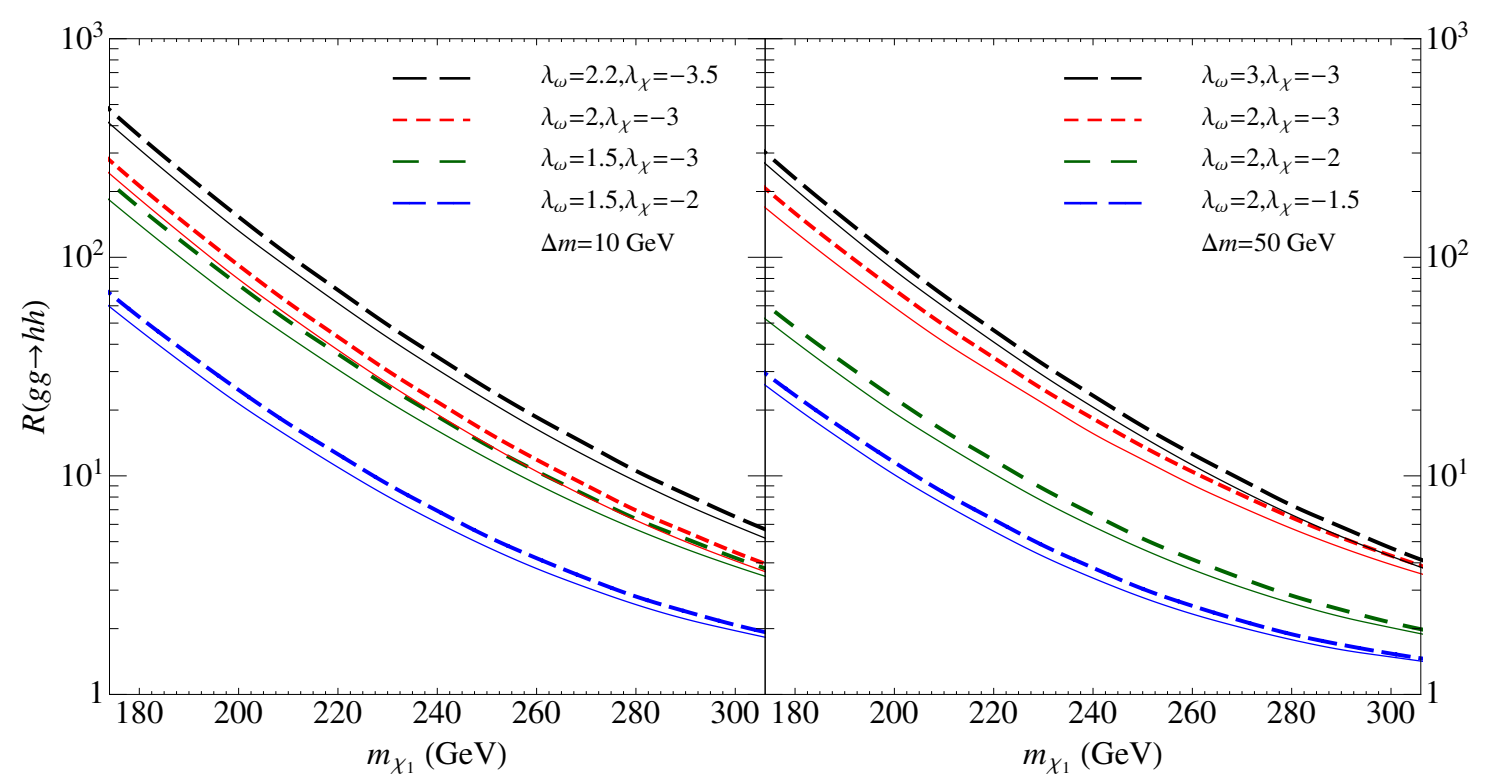

Figure 4. The ratio of Higgs pair productions due LQs and the SM for positive $\lambda_{\omega}$ and negative $\lambda_{\chi}$.

Therefore, taking $\sigma(p p \rightarrow t \bar{t} h) \simeq 80 \mathrm{fb}$ at LO and $B R(h \rightarrow \tau \tau) \simeq 7 \%$, one may conclude that $\sigma(p p \rightarrow L Q \bar{L} Q)$ should not exceed a few hundred fb to $O(1)$ pb at most. The exact constraint and implication of this process needs a thorough analysis and I do not attempt such study in this paper. 


\section{Conclusions and discussions}

The discovery of a scalar particle by CMS and ATLAS experiments at the LHC which appears to be essentially consistent with the SM Higgs picture within experimental margin of error is a triumph in our understanding of the fundamental dynamics. Undoubtedly, more precise measurements of the various production and decay channels are needed to nail down it as the Higgs of the SM. On the other hand, the confirmation itself still leave many questions unanswered which can be addressed with new dynamics or particles at the TEV range.

Colored particles around $\mathrm{TeV}$ scale have been studied in context of different theories for various reasons. With current 7 of $5 \mathrm{fb}^{-1}$ and $8 \mathrm{TeV}$ of $19.4 \mathrm{fb}^{-1}$ data, these can be probed if they are not too heavy. Among these the colored particles interacting with the SM Higgs doublet may cause an enhancement for Higgs pair production.

In the present paper, I have considered a several scalar LQs in which their portal couplings are such that its effect on the single Higgs production is within the limits given by the either CMS or ATLAS experiment. Even in this case it has been found that the Higgs pair production can be modified substantially. For several set of values for the portal couplings it has been shown that the rate may reach one to two orders magnitude higher than what it is in the SM. The two portal couplings are chosen to have an opposite sign which give reasonable single Higgs production rate via gluon fusion.

These are done via the following procedure. Upon scanning over these couplings for a low mass value the allowed regions by the Higgs porduction and decay to diphoton and $Z Z^{*}$ are obtained. Several set of values are chosen from these regions. We ignore the possibility of having both portal couplings are negative such that it produces a contribution twice as big as the SM one but with opposite sign. For the chosen values for the couplings the single Higgs productions have been plotted for masses upto $300 \mathrm{GeV}$ where the rates remain within the experimentally allowed region. Once this established, the Higgs pair production has been studied. For all the values the rates have been found to be enhanced by various values. For the sets with larger values, it may reach two orders of magnitude at lower range of LQ masses with moderate effect on the single Higgs production.

The effect becomes negligible above around the mass of $300 \mathrm{GeV}$. For this value, the enhancements range from few to at most an order of magnitude. In this case we have to wait the $14 \mathrm{TeV}$ run of the LHC experiments and high luminosity. Then the LQ will be ruled out or discovered before we reach the Higgs pair production discovery.

The present work demonstrates that the light colored particles with large portal couplings may reveal additional dynamics in the scalar potential. These are interesting due to their potential role in EWSB itself or in the thermal phase transition in the early universe. The model considered here is an example. From this study, one can see that any models with several color colored particles with strong couplings to Higgs can have sustantial effect on the Higgs pair production. 


\section{A Amplitudes}

Here we collect the formulae we used in our numerical calculations for the single and pair Higgs productions. The loop functions in eq. (3.3) for the single Higgs productions are give by:

$$
\begin{aligned}
& A_{1}(x)=-(2+3 x+3 x(2-x) f(x)) \text {, } \\
& A_{1 / 2}=2 x(1+(1-x) f(x)) \text {, } \\
& A_{0}=-x(1-x f(x)) \text {, } \\
& f(x)=\left\{\begin{array}{cl}
\arcsin ^{2}(1 / \sqrt{x}), & \text { if } x \geq 1 \\
-\frac{1}{4}\left(\log \frac{1+\sqrt{1-x}}{1-\sqrt{1-x}}-i \pi\right)^{2}, & \text { if } x<1 .
\end{array}\right.
\end{aligned}
$$

The Higgs pair production amplitudes are separated into two parts $F$ and $G$ from the same and opposite initial gluon polarizations respectively. The contributions from the SM for the process $g\left(p_{A}\right) g\left(p_{B}\right) \rightarrow h\left(p_{C}\right) h\left(p_{D}\right)$ are given by:

$$
\begin{aligned}
F_{\text {tri }}= & \frac{2 m_{t}^{2}}{s}\left(2+\left(4 m_{t}^{2}-s\right) C_{A B}\right) \\
F_{\text {box }}= & \frac{2 m_{t}^{2}}{s}\left(2+4 m_{t}^{2} C_{A B}-\left(s+2 m_{h}^{2}-8 m_{t}^{2}\right) m_{t}^{2}\left(D_{A B C}+D_{B A C}+D_{A C B}\right)\right. \\
& +\frac{m_{h}^{2}-4 m_{t}^{2}}{s}\left(\left(t-m_{h}^{2}\right)\left(C_{A C}+C_{B D}\right)+\left(u-m_{h}^{2}\right)\left(C_{B C}+C_{A D}\right)\right. \\
& \left.\left.-\left(t u-m_{h}^{4}\right) D_{A C B}\right)\right) \\
G_{\text {box }}= & \frac{m_{t}^{4}}{s\left(t u-m_{h}^{4}\right)}\left(\frac{\left(t^{2}+m_{h}^{4}-8 t m_{t}^{2}\right)}{m_{t}^{2}}\left(s C_{A B}+\left(t-m_{h}^{2}\right)\left(C_{A C}+C_{B D}\right)-s t D_{B A C}\right)\right. \\
& +\frac{\left(u^{2}+m_{h}^{4}-8 u m_{t}^{2}\right)}{m_{t}^{2}}\left(s C_{A B}+\left(u-m_{h}^{2}\right)\left(C_{B C}+C_{A D}\right)-s u D_{A B C}\right) \\
& -\frac{\left(t^{2}+u^{2}-2 m_{h}^{4}\right)\left(t+u-8 m_{t}^{2}\right)}{m_{t}^{2}} C_{C D} \\
& \left.-2\left(t+u-8 m_{t}^{2}\right)\left(t u-m_{h}^{4}\right)\left(D_{A B C}+D_{B A C}+D_{A C B}\right)\right) .
\end{aligned}
$$

Additional colored scalar particles contribute the following amplitudes:

$$
\begin{aligned}
F_{\text {tri }}^{S}= & -\frac{\lambda_{S} C_{s} v^{2}}{m_{S}^{2}}\left(2 m_{S}^{2} C_{A B}+1\right) \\
F_{\text {box }}^{S}= & -\frac{\lambda_{S} C_{s} v^{2}}{m_{S}^{2}}\left(2 m_{S}^{2} C_{A B}+1\right)-\frac{2 C_{s}\left(\lambda_{S} v^{2}\right)^{2}}{s}\left(m_{S}^{2}\left(D_{A B C}+D_{B A C}+D_{A C B}\right)\right. \\
& \left.-\frac{t-m_{h}^{2}}{s} C_{A C}-\frac{u-m_{h}^{2}}{s} C_{B C}+\frac{u t-m_{h}^{4}}{2 s} D_{A C B}\right)
\end{aligned}
$$




$$
\begin{aligned}
G_{\text {box }}^{S}= & -\frac{2 C_{s}\left(\lambda_{S} v^{2}\right)^{2}}{s}\left(m_{S}^{2}\left(D_{A B C}+D_{B A C}+D_{A C B}\right)-C_{C D}\right. \\
& +\frac{1}{2\left(t u-m_{h}^{4}\right)}\left(s t^{2} D_{B A C}+s u^{2} D_{A B C}\right. \\
& +s\left(s-2 m_{h}^{2}\right) C_{A B}+s\left(s-4 m_{h}^{2}\right) C_{C D} \\
& \left.\left.-2 t\left(t-m_{h}^{2}\right) C_{A C}-2 u\left(u-m_{h}^{2}\right) C_{B C}\right)\right) .
\end{aligned}
$$

Here $C_{A B}$ and $D_{A B C}$ etc are Passarino-Veltman 3 and 4-point functions and are given by

$$
\begin{aligned}
C_{A B} \equiv & \int \frac{d^{4} q}{i \pi} \frac{1}{\left(q^{2}-m^{2}\right)\left(\left(q+p_{A}\right)^{2}-m^{2}\right)\left(\left(q+p_{A}+p_{B}\right)^{2}-m^{2}\right)}, \\
D_{A B C} \equiv & \int \frac{d^{4} q}{i \pi} \frac{1}{\left(q^{2}-m^{2}\right)\left(\left(q+p_{A}\right)^{2}-m^{2}\right)\left(\left(q+p_{A}+p_{B}\right)^{2}-m^{2}\right)} \\
& \times \frac{1}{\left(\left(q+p_{A}+p_{B}+p_{C}\right)^{2}-m^{2}\right)} .
\end{aligned}
$$

Here $m=m_{t}$ and $m_{S}$ substitutions should be used for the top quark and colored scalar contribution respectively.

\section{Acknowledgments}

The author would like to thank Prof. Kai-Feng Chen for numerous discussions regarding the search of LQs at LHC and the authors of ref. [13] for providing their MadGraph implementation of the Higgs pair production for the SM and explaining the details. The travel support from The Mongolian Fund for Science and Technology and the local support from the organizers of the PASCOS 2013, where the work is presented, are acknowledged. The work was supported in part by the National Science Council of Taiwan under Grant No. NSC 101-2811-M-002-028.

Open Access. This article is distributed under the terms of the Creative Commons Attribution License (CC-BY 4.0), which permits any use, distribution and reproduction in any medium, provided the original author(s) and source are credited.

\section{References}

[1] ATLAS collaboration, Observation of a new particle in the search for the Standard Model Higgs boson with the ATLAS detector at the LHC, Phys. Lett. B 716 (2012) 1 [arXiv: 1207.7214] [INSPIRE].

[2] CMS collaboration, Observation of a new boson at a mass of $125 \mathrm{GeV}$ with the CMS experiment at the LHC, Phys. Lett. B 716 (2012) 30 [arXiv:1207.7235] [INSPIRE].

[3] O.J. Eboli, G. Marques, S. Novaes and A. Natale, Twin Higgs boson production, Phys. Lett. B 197 (1987) 269 [INSPIRE].

[4] E.N. Glover and J. van der Bij, Higgs boson pair production via gluon fusion, Nucl. Phys. B 309 (1988) 282 [INSPIRE]. 
[5] D.A. Dicus, C. Kao and S.S. Willenbrock, Higgs Boson Pair Production From Gluon Fusion, Phys. Lett. B 203 (1988) 457 [INSPIRE].

[6] T. Plehn, M. Spira and P. Zerwas, Pair production of neutral Higgs particles in gluon-gluon collisions, Nucl. Phys. B 479 (1996) 46 [Erratum ibid. B 531 (1998) 655] [hep-ph/9603205] [INSPIRE].

[7] S. Dawson, S. Dittmaier and M. Spira, Neutral Higgs boson pair production at hadron colliders: QCD corrections, Phys. Rev. D 58 (1998) 115012 [hep-ph/9805244] [INSPIRE].

[8] A. Djouadi, W. Kilian, M. Muhlleitner and P. Zerwas, Production of neutral Higgs boson pairs at LHC, Eur. Phys. J. C 10 (1999) 45 [hep-ph/9904287] [INSPIRE].

[9] LHC Higgs Cross section Working Group collaboration, S. Dittmaier et al., Handbook of LHC Higgs Cross sections: 1. Inclusive Observables, arXiv:1101.0593 [INSPIRE].

[10] G. Branco, P. Ferreira, L. Lavoura, M. Rebelo, M. Sher and J.P. Silva, Theory and phenomenology of two-Higgs-doublet models, Phys. Rept. 516 (2012) 1 [arXiv:1106.0034] [INSPIRE].

[11] J. Baglio, A. Djouadi, R. Gröber, M. Mühlleitner, J. Quevillon and M. Spira, The measurement of the Higgs self-coupling at the LHC: theoretical status, JHEP 04 (2013) 151 [arXiv: 1212.5581] [INSPIRE].

[12] M.J. Dolan, C. Englert and M. Spannowsky, Higgs self-coupling measurements at the LHC, JHEP 10 (2012) 112 [arXiv: 1206.5001] [INSPIRE].

[13] A. Papaefstathiou, L.L. Yang and J. Zurita, Higgs boson pair production at the LHC in the $b \bar{b} W^{+} W^{-}$channel, Phys. Rev. D 87 (2013) 011301 [arXiv:1209.1489] [InSPIRE].

[14] F. Goertz, A. Papaefstathiou, L.L. Yang and J. Zurita, Higgs Boson self-coupling measurements using ratios of cross sections, JHEP 06 (2013) 016 [arXiv:1301.3492] [INSPIRE].

[15] D. de Florian and J. Mazzitelli, Two-loop virtual corrections to Higgs pair production, Phys. Lett. B 724 (2013) 306 [arXiv:1305.5206] [INSPIRE].

[16] D. de Florian and J. Mazzitelli, Higgs Boson Pair Production at Next-to-Next-to-Leading Order in QCD, Phys. Rev. Lett. 111, 201801 (2013) 201801 [arXiv:1309.6594] [inSPIRE].

[17] J. Grigo, J. Hoff, K. Melnikov and M. Steinhauser, On the Higgs boson pair production at the LHC, Nucl. Phys. B 875 (2013) 1 [arXiv:1305.7340] [INSPIRE].

[18] V. Barger, L.L. Everett, C. Jackson and G. Shaughnessy, Higgs-Pair Production and Measurement of the Triscalar Coupling at LHC(8,14), arXiv:1311.2931 [INSPIRE].

[19] A. Belyaev, M. Drees, O.J. Eboli, J. Mizukoshi and S. Novaes, Supersymmetric Higgs pair production at hadron colliders, Phys. Rev. D 60 (1999) 075008 [hep-ph/9905266] [INSPIRE].

[20] E. Asakawa, D. Harada, S. Kanemura, Y. Okada and K. Tsumura, Higgs boson pair production in new physics models at hadron, lepton and photon colliders, Phys. Rev. D 82 (2010) 115002 [arXiv: 1009.4670] [INSPIRE].

[21] G.D. Kribs and A. Martin, Enhanced di-Higgs Production through Light Colored Scalars, Phys. Rev. D 86 (2012) 095023 [arXiv:1207.4496] [inSPIRE].

[22] I. Dorsner, S. Fajfer, A. Greljo and J.F. Kamenik, Higgs Uncovering Light Scalar Remnants of High Scale Matter Unification, JHEP 11 (2012) 130 [arXiv:1208.1266] [INSPIRE].

[23] J. Cao, Z. Heng, L. Shang, P. Wan and J.M. Yang, Pair Production of a $125 \mathrm{GeV}$ Higgs Boson in MSSM and NMSSM at the LHC, JHEP 04 (2013) 134 [arXiv:1301.6437] [INSPIRE]. 
[24] Z. Heng, L. Shang and P. Wan, Pair production of a 125 GeV Higgs boson in MSSM and NMSSM at the ILC, JHEP 10 (2013) 047 [arXiv: 1306.0279] [INSPIRE].

[25] C. Han, X. Ji, L. Wu, P. Wu and J.M. Yang, Higgs pair production with SUSY QCD correction: revisited under current experimental constraints, arXiv:1307.3790 [INSPIRE].

[26] L. Wang, W. Wang, J.M. Yang and H. Zhang, Higgs-pair production in littlest Higgs model with T-parity, Phys. Rev. D 76 (2007) 017702 [arXiv:0705.3392] [INSPIRE].

[27] X.-F. Han, L. Wang and J.M. Yang, Higgs-pair Production and Decay in Simplest Little Higgs Model, Nucl. Phys. B 825 (2010) 222 [arXiv:0908.1827] [InSPIRE].

[28] M.J. Dolan, C. Englert and M. Spannowsky, New Physics in LHC Higgs boson pair production, Phys. Rev. D 87 (2013) 055002 [arXiv:1210.8166] [INSPIRE].

[29] H. Sun and Y.-J. Zhou, Enhancement of Loop induced 125GeV Higgs pair production through Large-Extra-Dimensions model at the LHC, JHEP 11 (2012) 127 [arXiv:1211.6201] [INSPIRE].

[30] L. Wang and X.-F. Han, LHC diphoton Higgs signal and top quark forward-backward asymmetry in quasi-inert Higgs doublet model, JHEP 05 (2012) 088 [arXiv: 1203.4477] [INSPIRE].

[31] D.Y. Shao, C.S. Li, H.T. Li and J. Wang, Threshold resummation effects in Higgs boson pair production at the $L H C$, arXiv: 1301.1245 [INSPIRE].

[32] J.M. No and M. Ramsey-Musolf, Probing the Higgs Portal at the LHC Through Resonant di-Higgs Production, arXiv:1310.6035 [INSPIRE].

[33] N. Haba, K. Kaneta, Y. Mimura and E. Tsedenbaljir, Higgs Pair Production at the LHC and ILC from general potential, arXiv:1311.0067 [INSPIRE].

[34] H. Georgi and S. Glashow, Unity of All Elementary Particle Forces, Phys. Rev. Lett. 32 (1974) 438 [INSPIRE].

[35] J.C. Pati and A. Salam, Lepton Number as the Fourth Color, Phys. Rev. D 10 (1974) 275 [Erratum ibid. D 11 (1975) 703-703] [INSPIRE].

[36] G. Senjanović and A. Sokorac, Light Leptoquarks in SO(10), Z. Phys. C 20 (1983) 255 [INSPIRE].

[37] O.U. Shanker, $\pi \ell$ 2, $K \ell 3$ and $K^{0}-\bar{K} 0$ Constraints on Leptoquarks and Supersymmetric Particles, Nucl. Phys. B 204 (1982) 375 [InSPIRE].

[38] W. Buchmüller and D. Wyler, Constraints on SU(5) Type Leptoquarks, Phys. Lett. B 177 (1986) 377 [INSPIRE].

[39] W. Buchmüller, R. Ruckl and D. Wyler, Leptoquarks in lepton-quark collisions, Phys. Lett. B 191 (1987) 442 [Erratum ibid. B 448 (1999) 320] [INSPIRE].

[40] V. Angelopoulos, J.R. Ellis, H. Kowalski, D.V. Nanopoulos, N. Tracas and F. Zwirner, Search for New Quarks Suggested by the Superstring, Nucl. Phys. B 292 (1987) 59 [InSPIRE].

[41] J.L. Hewett and T.G. Rizzo, Low-Energy Phenomenology of Superstring Inspired E 6 Models, Phys. Rept. 183 (1989) 193 [INSPIRE].

[42] S. Dimopoulos and L. Susskind, Mass Without Scalars, Nucl. Phys. B 155 (1979) 237 [INSPIRE].

[43] S. Dimopoulos, Technicolored Signatures, Nucl. Phys. B 168 (1980) 69 [inSPIRE].

[44] E. Eichten and K.D. Lane, Dynamical Breaking of Weak Interaction Symmetries, Phys. Lett. B 90 (1980) 125 [INSPIRE]. 
[45] E. Farhi and L. Susskind, Technicolor, Phys. Rept. 74 (1981) 277 [InSPIRE].

[46] B. Schrempp and F. Schrempp, Light Leptoquarks, Phys. Lett. B 153 (1985) 101 [INSPIRE].

[47] J. Wudka, Composite Leptoquarks, Phys. Lett. B 167 (1986) 337 [INSPIRE].

[48] B. Gripaios, Composite Leptoquarks at the LHC, JHEP 02 (2010) 045 [arXiv:0910.1789] [INSPIRE].

[49] P. Hung and C. Xiong, Renormalization Group Fixed Point with a Fourth Generation: Higgs-induced Bound States and Condensates, Nucl. Phys. B 847 (2011) 160 [arXiv:0911.3890] [INSPIRE].

[50] T. Enkhbat, W.-S. Hou and H. Yokoya, Early LHC Phenomenology of Yukawa-bound Heavy $Q \bar{Q}$ Mesons, Phys. Rev. D 84 (2011) 094013 [arXiv: 1109.3382] [InSPIRE].

[51] K. Babu and J. Julio, Two-Loop Neutrino Mass Generation through Leptoquarks, Nucl. Phys. B 841 (2010) 130 [arXiv: 1006.1092] [INSPIRE].

[52] K. Babu and J. Julio, Radiative Neutrino Mass Generation through Vector-like Quarks, Phys. Rev. D 85 (2012) 073005 [arXiv:1112.5452] [INSPIRE].

[53] M. Kohda, H. Sugiyama and K. Tsumura, Lepton number violation at the LHC with leptoquark and diquark, Phys. Lett. B 718 (2013) 1436 [arXiv:1210.5622] [INSPIRE].

[54] M. Pietroni, The electroweak phase transition in a nonminimal supersymmetric model, Nucl. Phys. B 402 (1993) 27 [hep-ph/9207227] [INSPIRE].

[55] J.M. Cline and G.D. Moore, Supersymmetric electroweak phase transition: Baryogenesis versus experimental constraints, Phys. Rev. Lett. 81 (1998) 3315 [hep-ph/9806354] [INSPIRE].

[56] J.M. Cline, G.D. Moore and G. Servant, Was the electroweak phase transition preceded by a color broken phase?, Phys. Rev. D 60 (1999) 105035 [hep-ph/9902220] [INSPIRE].

[57] M. Carena, G. Nardini, M. Quirós and C. Wagner, The Baryogenesis Window in the MSSM, Nucl. Phys. B 812 (2009) 243 [arXiv:0809.3760] [INSPIRE].

[58] T. Cohen and A. Pierce, Electroweak Baryogenesis and Colored Scalars, Phys. Rev. D 85 (2012) 033006 [arXiv:1110.0482] [INSPIRE].

[59] D.J. Chung, A.J. Long and L.-T. Wang, The 125 GeV Higgs and Electroweak Phase Transition Model Classes, Phys. Rev. D 87 (2013) 023509 [arXiv:1209.1819] [InSPIRE].

[60] W. Huang, J. Shu and Y. Zhang, On the Higgs Fit and Electroweak Phase Transition, JHEP 03 (2013) 164 [arXiv: 1210.0906] [INSPIRE].

[61] M. Laine, G. Nardini and K. Rummukainen, Lattice study of an electroweak phase transition at $m_{h} 126 \mathrm{GeV}, J C A P 01$ (2013) 011 [arXiv:1211.7344] [INSPIRE].

[62] H.H. Patel, M.J. Ramsey-Musolf and M.B. Wise, Color Breaking in the Early Universe, Phys. Rev. D 88 (2013) 015003 [arXiv:1303.1140] [InSPIRE].

[63] CMS collaboration, Search for pair production of first- and second-generation scalar leptoquarks in pp collisions at $\sqrt{s}=7 \mathrm{TeV}$, Phys. Rev. D 86 (2012) 052013 [arXiv: 1207.5406] [INSPIRE].

[64] CMS collaboration, Search for Pair-production of Second generation Leptoquarks in $8 \mathrm{TeV}$ proton-proton collisions, CMS-PAS-EXO-12-042.

[65] CMS collaboration, Search for third-generation leptoquarks and scalar bottom quarks in pp collisions at $\sqrt{s}=7 \mathrm{TeV}$, JHEP 12 (2012) 055 [arXiv:1210.5627] [INSPIRE]. 
[66] CMS collaboration, Search for pair production of third-generation leptoquarks and top squarks in pp collisions at $\sqrt{s}=7$ TeV, Phys. Rev. Lett. 110 (2013) 081801 [arXiv:1210.5629] [INSPIRE].

[67] ATLAS collaboration, Search for third generation scalar leptoquarks in pp collisions at $\sqrt{s}=$ $7 \mathrm{TeV}$ with the ATLAS detector, JHEP 06 (2013) 033 [arXiv:1303.0526] [INSPIRE].

[68] ATLAS collaboration, Search for 1st-Generation Leptoquarks Using the ATLAS Detector, EPJ Web Conf. 28 (2012) 12012 [arXiv:1202.1369] [INSPIRE].

[69] ATLAS collaboration, Search for second generation scalar leptoquarks in pp collisions at $\sqrt{s}=7$ TeV with the ATLAS detector, Eur. Phys. J. C 72 (2012) 2151 [arXiv:1203.3172] [INSPIRE].

[70] ATLAS collaboration, Measurements of Higgs boson production and couplings in diboson final states with the ATLAS detector at the LHC, Phys. Lett. B 726 (2013) 88 [arXiv: 1307.1427] [INSPIRE].

[71] CMS collaboration, Updated measurements of the Higgs boson at 125 GeV in the two photon decay channel, CMS-PAS-HIG-13-001.

[72] CMS collaboration, Properties of the Higgs-like boson in the decay H to ZZ to $4 \mathrm{l}$ in $\mathrm{pp}$ collisions at $\sqrt{s}=7$ and 8 TeV, CMS-PAS-HIG-13-002.

[73] M. Krämer, T. Plehn, M. Spira and P. Zerwas, Pair production of scalar leptoquarks at the CERN LHC, Phys. Rev. D 71 (2005) 057503 [hep-ph/0411038] [INSPIRE].

[74] A. Belyaev, C. Leroy, R. Mehdiyev and A. Pukhov, Leptoquark single and pair production at LHC with CalcHEP/CompHEP in the complete model, JHEP 09 (2005) 005 [hep-ph/0502067] [INSPIRE].

[75] B. Gripaios, A. Papaefstathiou, K. Sakurai and B. Webber, Searching for third-generation composite leptoquarks at the LHC, JHEP 01 (2011) 156 [arXiv:1010.3962] [INSPIRE].

[76] S. Davidson and P. Verdier, Leptoquarks decaying to a top quark and a charged lepton at hadron colliders, Phys. Rev. D 83 (2011) 115016 [arXiv:1102.4562] [InSPIRE].

[77] Particle Data Group collaboration, J. Beringer et al., Review of Particle Physics (RPP), Phys. Rev. D 86 (2012) 010001 [InSPIRE].

[78] S. Davidson, D.C. Bailey and B.A. Campbell, Model independent constraints on leptoquarks from rare processes, Z. Phys. C 61 (1994) 613 [hep-ph/9309310] [INSPIRE].

[79] S. Davidson and S. Descotes-Genon, Minimal Flavour Violation for Leptoquarks, JHEP 11 (2010) 073 [arXiv: 1009.1998] [INSPIRE].

[80] M. Reece, Vacuum Instabilities with a Wrong-Sign Higgs-Gluon-Gluon Amplitude, New J. Phys. 15 (2013) 043003 [arXiv: 1208.1765] [INSPIRE].

[81] J. Alwall, M. Herquet, F. Maltoni, O. Mattelaer and T. Stelzer, MadGraph 5: Going Beyond, JHEP 06 (2011) 128 [arXiv:1106.0522] [INSPIRE].

[82] T. Sjöstrand, S. Mrenna and P.Z. Skands, PYTHIA 6.4 Physics and Manual, JHEP 05 (2006) 026 [hep-ph/0603175] [INSPIRE].

[83] CMS collaboration, Search for Higgs Boson Production in Association with a Top-Quark Pair and Decaying to Bottom Quarks or Tau Leptons, CMS-PAS-HIG-13-019.

[84] CMS collaboration, Search for the standard model Higgs boson produced in association with top quarks in multilepton final states, CMS-PAS-HIG-13-020. 